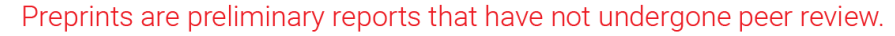 They should not be considered conclusive, used to inform clinical practice, or referenced by the media as validated information. \\ Defining the true hardness of materials harder than single crystal diamond
}

Guodong (David) Zhan ( $\sim$ guodong.zhan@aramco.com )

Saudi Aramco (Saudi Arabia)

Jin Liu

Sichuan University

Pei Wang

High Pressure Science and Engineering Center, University of Nevada

\section{Liping Wang}

Southern University of Science and Technology https://orcid.org/0000-0002-6137-3113

\section{Xiaozhi Yan}

High Pressure Science and Engineering Center, University of Nevada

\section{Yongtao Zou}

Shenzhen Technology University

\section{Duanwei He}

Sichuan University

\section{Chinthaka Gooneratne}

Saudi Aramco (Saudi Arabia)

Jianhui Xu

Saudi Aramco

\section{Alawi Alalsayednassir}

Saudi Aramco (Saudi Arabia)

\section{Timothy Moellendick}

Saudi Aramco (Saudi Arabia)

\section{Article}

Keywords: diamond, superhard materials, Vickers Hardness Tester

Posted Date: June 25th, 2021

DOI: https://doi.org/10.21203/rs.3.rs-614606/v1

License: (9) (1) This work is licensed under a Creative Commons Attribution 4.0 International License. Read Full License 



\title{
Defining the true hardness of materials harder than single crystal diamond
}

Jin Liu ${ }^{1,2,3}$, Guodong (David) Zhan ${ }^{4 *}$, Pei Wang ${ }^{3}$, Liping Wang ${ }^{3 *}$, Xiaozhi Yan ${ }^{3}$, Yongtao Zou ${ }^{5}$, Duanwei $\mathrm{He}^{1^{*}}$, Chinthaka P. Gooneratne ${ }^{4}$, Jianhui $\mathrm{Xu}^{4}$, Alawi G Alalsayednassir, ${ }^{6}$ Timothy Eric Moellendick $^{4}$

\section{Affiliations:}

${ }^{1}$ Institute of Atomic and Molecular Physics, Sichuan University; Chengdu 610065, People's Republic of China.

${ }^{2}$ School of Mechanical Engineering, Jingchu University of Technology; Jingmen 44800, People's Republic of China.

${ }^{3}$ Academy for Advanced Interdisciplinary Studies, and Department of Physics, Southern University of Science and Technology; Shenzhen 518055, People's Republic of China.

${ }^{4}$ Drilling Technology Division, Exploration and Petroleum Engineering - Advanced Research Center; Saudi Aramco, Dhahran 31311, Saudi Arabia.

${ }^{5}$ College of Engineering Physics, and Center for Advanced Material Diagnostic Technology, Shenzhen Technology University; Shenzhen 518118, People's Republic of China.

${ }^{6}$ Drilling Technical Department, Drilling and Workwork (D\&WO), Saudi Aramco, Dhahran, 31311, Saudi Arabia.

*Corresponding authors. Emails: guodong.zhan@aramco.com; wanglp3@ sustech.edu.cn; duanweihe@scu.edu.cn

\begin{abstract}
With the development of new synthesis methods and chemistries, a number of new superhard materials have been reported to be harder than diamond. While such materials are highly desirable due to their wide-ranging applications, there are some inherent uncertainties in the methods utilized to determine and define the hardness of such materials. In this paper, we employed the Vickers Hardness Tester to measure the hardness of nine ceramic and superhard materials within a well-defined criteria and methodology, for the reliable assessment of the hardness of these new superhard materials. These findings and the developed testing method have broad implications in the characterizing of new and emerging superhard materials, leading to new discoveries.
\end{abstract}

\section{Introduction}

Hardness is one of the most important mechanical properties of materials, especially for hard and superhard materials, and is often utilized to determine their applications ${ }^{1-5}$. The Vickers or Knoop hardness testers are the most traditional and common methods of measuring the hardness 
of materials, where square-based pyramid indenter tips made of natural single crystal diamond (N-D) makes an indent on the material. Recently, Vickers hardness values between 120 and 200 GPa have been obtained for newly developed materials utilizing standard N-D indenters ${ }^{6,7,8}$. In fact, due to the anisotropy in the properties of N-D, the reported hardness values ranged from 60 to $120 \mathrm{GPa}$ depending on the crystal orientation ${ }^{9-14}$. When preparing the N-D indenter tip, the direction perpendicular to the (111) crystal plane is always carefully selected as the axis of the indenter to exploit its highest hardness. For low-hardness materials, the hardness measurement results can be easily calculated and interpreted by classical equations and methods ${ }^{15-18}$. However, uncertainties arise when the hardness of the tested material is close to or greater than that of the $\mathrm{N}-\mathrm{D}$ indenter, leading to challenges in reliably evaluating the hardness of materials. The work in reference ${ }^{19}$ indicated that the testing parameters should be taken into account when measuring hardness values of materials harder than diamond. However, there are still valid concerns about the inconsistency of hardness values from one test to another, due to the possible permanent plastic deformation or damage of the N-D indenter after conducting a test. Moreover, debates have lingered for well over decades on 'if superhard materials are really harder than diamond' and how the hardness of such new superhard materials are incorrect and misleading due to a lack of clarity on testing procedure ${ }^{20}$. Therefore, in this day and age of rapid advances in material science, it is worthwhile to investigate the true nature of superhard materials and develop a proper, usable framework to determine hardness of such materials. In this work, we study the applicability of the Vickers hardness indentation method to superhard materials in terms of reliability and consistency. To identify the effect the hardness differences between indenters and tested materials has on the measured hardness values, we developed a systematic and repeatable criterion to reliably assess the hardness of new or emerging materials, for the first time compared to known literature. The criterion provides insights into the hardness assessment of the new materials harder than diamond, and can play a critical role to advance new material development and engineering applications.

\section{Results}

Framework for defining true hardness. The hardness of a material is a measure of its ability to resist deformation upon a load under isostatic pressure. The Vickers indenter is a regular pyramid typically made of natural single crystal diamond with an angle of $136^{\circ}$ between the opposite faces. During the measurement, the diamond pyramid is pressed into the material to be tested under a pre-determined load and dwell time. After unloading, a quadrangle indentation on the tested material, resulting from localized plastic deformation caused by the indenter, is observed under a microscope. Fig. 1 schematically shows the cross-section of the pyramidal indenter in axial direction, the relative interaction between the indenter and a tested material, and the produced square indentation in a tested material. Details about the preparation of indenter tips and tested samples from three families of representative materials, ceramic materials, superhard materials, and single crystal diamonds (natural and synthetic), are in (21). As shown in Figs. 1 (b) and (c), we can get the Vickers hardness (HV) value after measuring the lengths of two diagonal lines $\mathrm{d} 1$ and $\mathrm{d} 2$ using equation:

$$
H_{V}=1854.4 \frac{P}{d^{2}}
$$


where $\mathrm{P}$ is the loading force in grams, and $\mathrm{d}$ is the arithmetic mean of the two diagonals of the indentation $\mathrm{d} 1$ and $\mathrm{d} 2$ in micrometers (22). In fact, hardness measurements are closely related to the material of Vickers indenter, applying load, dwell time, polishing quality of the sample surface, crystal orientation of the sample, etc. Six materials including $\mathrm{Al}_{2} \mathrm{O}_{3}, \mathrm{SiC}_{2} \mathrm{PcBN}, \mathrm{PCD}$, $\mathrm{N}-\mathrm{D}$, and SC-D with various hardness are selected and prepared for the Vickers indenter tips.

In order to study the influence of the hardness of the Vickers indenter tip material on the measurement reliability of the tested material, a new evaluation method is proposed in this paper. Here are the definitions of the measurements: $H_{I}$, the hardness value of the prepared indenter tested by standard N-D Vickers diamond indenter; $H_{S}$, the hardness value of tested sample by the standard N-D Vickers diamond indenter; $H_{O}$, the measured hardness value of tested sample by the prepared indenter. Two normalized equations for these corresponding measurements of $H_{I}$, $H_{S}$ and $H_{O}$ are shown below:

$$
\begin{gathered}
\Delta H=\frac{H_{I}-H_{S}}{H_{S}} \times 100 \% \\
\Delta H_{O}=\frac{H_{o}-H_{S}}{H_{S}} \times 100 \%
\end{gathered}
$$

where $\Delta H$ is the percentage of the hardness difference between the prepared indenter and tested sample relative to hardness of the tested sample; $\Delta H_{O}$ is the percentage of hardness difference between the measured value and value of the tested sample relative to the hardness of tested sample. In order to achieve the reliable hardness result, its corresponding $\Delta H_{O}$ should be as low as possible, sometimes $<5 \%$.

Vicker's Hardness Tests. A series of experiments were performed based on the $\mathrm{H}_{\mathrm{v}}$ values of the samples (21). In the first sample group the test samples and the indenters are made of the same material, for example PcBN (indenter) vs PcBN (tested sample). For comparative observation and analysis of the indentations produced by different types of indenter materials, the indentations produced by the N-D indenter tip are marked with yellow arrows while those generated by the prepared indenter materials tips are marked with white arrows, as shown in Figs. 2. Moreover, representative SEM images of the indentations produced are shown in insert above (by standard N-D indenter) and insert below (by the prepared indenter) in Fig. 2. As an example of the first group, PcBN sample is tested by the standard N-D indenter and an PcBN indenter, with loads of 0.3 and $3 \mathrm{Kg}$. The sizes of indentations produced by PcBN indenter are obviously smaller than the corresponding indentations produced by standard N-D indenter using the same loads, as shown in Fig. 2 (a). The SEM images of indentations with a load of $3 \mathrm{~kg}$ is shown in the insert of Fig. 2 (a). The $H_{O}$ value of PcBN is $48.05 \mathrm{GPa}$, which is significantly greater than the $H_{I}$ value of $30.11 \mathrm{GPa}$ measured by the standard N-D indenter, resulting in an $\Delta H_{O}$ of $59.56 \%$. This indicates that the hardness measurement of the tested PcBN sample with 
the indenter made of the same material is unreliable. Similar results were observed for other experiments performed with the first group of materials (21).

In the second sample group, the test samples are harder than the indenters, for example SiC (indenter) vs PcBN (test sample). As an example of the second group, a PcBN sample was tested by a $\mathrm{SiC}$ indenter as shown in Fig. 2(b). The indentations produced by the $\mathrm{SiC}$ indenter were shown to be irregular quadrangles compared to those produced by standard a N-D indenter. The high-resolution SEM images showed clear indentations for a load of $3 \mathrm{~kg}$ - insert of Fig. 2(b). The $H_{O}$ value of $\mathrm{PcBN}$ is $47.5 \mathrm{GPa}$, which is significantly greater than the $H_{I}$ value of 30.11 $\mathrm{GPa}$, giving rise to a $\Delta \mathrm{H}$ of $-15.4 \%$ and a $\Delta H_{O}$ of $57.73 \%$. The high $\Delta H_{O}$ suggests that the hardness measurement value of PcBN (test sample) by the $\mathrm{SiC}$ (indenter) is unreliable.

In the third and final group, the test samples are softer than the indenters, for example PcBN (indenter) vs $\mathrm{SiC}$ (test sample). An example of experiments performed with a couple of group 3 materials is shown in Fig. 2 (c), where the hardness of SiC test sample was measured by a PcBN indenter. The $\mathrm{HO}$ value of $25.68 \mathrm{GPa}$, which is almost equal to $\mathrm{HI}$ value of $25.48 \mathrm{GPa}$, leads to a $\Delta \mathrm{H}$ of $18.2 \%$ and $\Delta \mathrm{HO}$ of $0.78 \%$. This clearly indicates that the hardness measurement of SiC by both PcBN (prepared indenter) and N-D indenters are reliable.

Reliability of hardness values. Figure 3 summarizes the experimental performed with all three groups and shows how the hardness differences between the test samples and indenters $(\Delta \mathrm{H})$ influence the reliability $\left(\Delta \mathrm{H}_{\mathrm{O}}\right)$ of hardness values. When $\Delta \mathrm{H}$ is less than or equal to zero, where the hardness of the indenter is equal to or less than the hardness of the test sample, the measured hardness value of the test sample is higher than the nominal value measured by the standard N-D indenter. The $\Delta \mathrm{H}_{\mathrm{O}}$ is always higher than $10 \%$ in the tests performed in this study, which indicates that the hardness values are unreliable (21). The reliability is estimated to be significantly higher if $\Delta \mathrm{H}$ is positive, meaning the hardness of the indenter is higher than the test sample. For the case of $\Delta H=18.2 \%$, when a SiC sample is tested by a PcBN indenter, the deviation of hardness from the nominal value tested by the standard N-D indenter was found to be only $0.78 \%$. In this study, when the $\Delta \mathrm{H}$ is equal to or higher than $18.2 \%, \Delta \mathrm{H}_{\mathrm{O}}$ is always less than $4.35 \%$, which indicates the measurement is reliable. The detailed statistical analysis and reliability results based on measured values $\Delta \mathrm{H}$ and $\Delta \mathrm{Ho}$ are shown in the Fig. 3 and (21). During the loading process of indentation hardness measurements, the indenter exerts a force on the surface of the test sample to generate high stress in the locally affected field with both elastic and plastic deformation. The indenter, on the other hand, ideally should not experience plastic deformation and is therefore required to be harder than the tested material. (23). However, when the test materials are harder than the tip of the indenter (19), the indenter will induce significant elastic and plastic deformation, or even brittle cracking, of the tip during the testing process leading to the measured value being unreliable.

\section{Discussion}

We prepared a series of indenters and test samples with known hardness, pre-characterized by the standard indenter made of natural single crystal diamond, and carried out Vickers hardness cross-tests to investigate the influence the hardness differences between the indenters and the tested samples has on the reliability of hardness measurements of a variety of superhard materials. We found that when the hardness of the Vickers indenter is close to or below the 
hardness of the sample to be tested, the hardness value measured by the Vickers indentation method is significantly higher than the nominal value. The hardness value of the tested sample, therefore, is severely overestimated. On the other hand, when the hardness of the indenter is $18.2 \%$ higher than that of the tested sample or more, we can say, with high confidence, that the results are sufficiently reliable to characterize and define superhard materials. Therefore, this study sets a standard on the method to test superhard materials that are developed to be harder than diamond, since hardness values measured by the Vickers hardness tests will only be reliable and accurate only when the indenters are $18.2 \%$ harder than newly-developed superhard materials. Most recently, we produced ultra-strong catalyst-free polycrystalline diamond that broke all single crystal diamond indenters during testing (24), a clear indication that it may be one of the world's hardest materials. Therefore, this developed material could be a promising Vickers indenter material to replace the currently used single crystal diamond, for the testing and characterization of newly-developed superhard materials.

\section{Methods}

Preparation of Vicker's indenters and tested samples. In order to accurately assess the applicability of Vickers hardness method, three families of representative materials, including ceramic materials, super-hard materials, and single crystal diamonds (natural and synthetic), were carefully selected for the preparation of Vickers hardness indenter tips and test samples (Fig. S1). The opposite intersection angle of square pyramid of indenter tip was $136^{\circ} \pm 15^{\prime}$ and the chisel edge was less than $1 \mu \mathrm{m}$ wide, which are the acknowledged and standard geometries of a Vickers indenter. All the prepared indenters and test samples were polished to a mirror finish as shown in Fig. S1. The ceramics family in this study include alumina (A12O3) and silicon carbide (SiC). The superhard materials family include polycrystalline cubic boron nitride (PcBN), polycrystalline diamond (PCD) and much harder diamond-cBN composites (BCNs). The diamonds family include natural single crystal diamond (N-D) and synthetic single crystal diamond (SC-D). Due to challenges in the preparation process and hardness measurement, N-D was selected only for the standard Vickers indenter tip with a direction of [111] crystal orientation as the axis of the indenter. Fig. S2 shows the experimental results for tests performed with an N-D indenter to obtain the Vickers hardness values for several test samples (Table S1). SC-D, on the other hand, was processed for utilization as a hardness indenter tip and test sample with [111] crystal orientation for both the axis of the indenter and the testing direction of the test sample (see Table S2 for more details). Two cemented tungsten carbide (WC-Co) hard metals, YG 6 (WC-Co with $6 \mathrm{wt} \%$ of cobalt) and YG 8 (WC-Co with $8 \mathrm{wt} \%$ cobalt), were utilized as test samples due to their extensive applications in various industries.

Characterization methods. The Vickers hardness of the polished samples was tested by the Vickers single crystal diamond tester (FV-700B, Future -Tech Corp. Japan), with different loads and same dwelling time of $15 \mathrm{~s}$. The morphologies, sizes and lengths of the indentations produced by standard N-D pyramidal indenter and prepared indenters were observed, measured 
and analyzed by the Vickers hardness tester (FV-700B, Future -Tech, Japan) and the scanning electron microscopy (SEM) (FEI INSPECT F50, USA).

\section{Data availability}

The data that support the findings of this study are available from the corresponding author upon reasonable request.

\section{References}

1. S. Veprek, in Handbook of Ceramic Hard Materials, (ed. by R. Riedel, Wiley, Weinheim, 2000), pp. 104-139.

2. J. Qin, D. He, J. Wang, L. Fang, L. Lei, Y. Li, J. Hu, Z. Kou, Y. Bi, Is rhenium diboride a superhard material? Adv. Mater. 20, 4780 (2008).

3. H. Sumiya, Y. Ishida, K. Arimoto, K. Harano, Real indentation hardness of nanopolycrystalline cBN synthesized by direct conversion sintering under HPHT, Diam. Relat. Mater. 48, 47 (2014).

4. B. Xu, Y. Tian, Ultrahardness: measurement and enhancement, J. Phys. Chem. C 119, 5633 (2015).

5. G. Ceder, Predicting properties from scratch, Science 280, 1099 (1998).

6. T. Irifune, A. Kurio, S. Sakamoto, T. Inoue, H. Sumiya, Ultrahard polycrystalline diamond from graphite, Nature 421, 599 (2003).

7. H. Sumiya, T. Irifune, Hardness and deformation microstructures of nano-polycrystalline diamonds synthesized from various carbons under high pressure and high temperature, $J$. Mater. Res. 22, 2345 (2007).

8. J. Xiao, B. Wen, B. Xu, X. Zhang, Y. Wang, Y. Tian, Intersectional nanotwinned diamond-the hardest polycrystalline diamond by design, NPJ Compu.Mats, 6, 1 (2020).

9. Q. Huang, D. Yu, B. Xu, W. Hu, Y. Ma, Y. Wang, Z. Zhao, B. Wen, J. He, Z. Liu, Y. Tian, 
Nanotwinned diamond with unprecedented hardness and stability, Nature 510, 250 (2014).

10. Y. Tian, B. Xu, D. Yu, Y. Ma, Y. Wang, Y. Jiang, W. Hu, C. Tang, Y. Gao, K. Luo, Z. Zhao, L. Wang, B. Wen, J. He, Z. Liu, Ultrahard nanotwinned cubic boron nitride, Nature 493, 385 (2013).

11. C. Yan, H. Mao, W. Li, J. Qian, Y. Zhao, R. Hemley, Ultrahard diamond single crystals from chemical vapor deposition, J. Status Solidi A 201, R25 (2004).

12. J. Liu, G. Zhan, Q. Wang, X. Yan, F. Liu, P. Wang, L. Lei, F. Peng, Z. Kou, D. He, Superstrong micro-grained polycrystalline diamond compact through work hardening under high pressure, Appl. Phys. Lett. 112, 061901 (2018).

13. N. Dubrovinskaia, S. Dub, L. Dubrovinsky, Superior wear resistance of aggregated diamond nanorods, Nano Lett. 6, 824 (2006).

14. J.E. Field, in The Properties of Natural and Synthetic Diamond (Academic, London, 1992).

15. A.R. Franco Jr, G. Pintaude, A. Sinatora, C.E. Pinedo, A.P. Tschiptschin, The use of a Vickers indenter in depth sensing indentation for measuring elastic modulus and Vickers hardness, Mater. Res. 7, 483 (2004).

16. R.Y. Lo, D.B. Bogy, Compensating for elastic deformation of the indenter in hardness tests of very hard materials, J. Mater. Res. 14, 2276 (1999).

17. W.C. Oliver, G.M. Pharr, An improved technique for determining hardness and elastic modulus using load and displacement sensing indentation experiments, J. Mater. Res. 7, 1564 (1992).

18. E. Atar, H. Cimenoglu, E.S. Kayali, Hardness characterization of thin $\mathrm{Zr}$ (Hf, N) coatings, Surf. Coat. Tech. 162, 167 (2003).

19. V. Brazhkin, N. Dubrovinskaia, M. Nicol, N. Novikov, R. Riedel, V. Solozhenko, Y. Zhao, What does' harder than diamond' mean?, Nat. Mater. 3, 576 (2004).

20. M. Chaudri, M. Munawar, Y.Y. Lim, Harder than diamond? Just fiction, Nat. Mater. 4, 4 (2005). 
21. Materials and methods are available as supplementary materials.

22. D. He, Y. Zhao, L. Daemen, J. Qian, T.D. Shen, T.W. Zerda, Boron suboxide: As hard as cubic boron nitride, Appl. Phys. Lett. 81, 643 (2002).

23. D. He, S.R. Shieh, T.S. Duffy, Strength and equation of state of boron suboxide from radial xray diffraction in a diamond cell under nonhydrostatic compression, Phys. Rev. B. 70, 184121 (2004).

24. Q. L, G. Zhan, D. Li, D. He, T.E. Moellendick, C.P. Gooneratne, A.G. Alalsayednassir, Ultrastrong catalyst-free polycrystalline diamond, Nat. Sci. Rep. 10, 22020 (2020).

Acknowledgments: The authors would like to thank the following funding sources. Funding:

National Key R\&D Program of China (No.2018YFA0305900)

National Natural Science Foundation of China (Grant Nos. 51472171, 11427810, 11872198 and 11704014)

Science and Technology Innovation Team of Sichuan Province (Grant No. 15CXTD0025)

Science and Technology Innovation Team of Jingchu University of technology (Grant No. TD201906)

Partially supported by Natural Science Foundation of Top Talent of SZTU to Y. T. Zou Collaborative project fund between Saudi Aramco and Chengdu Dongwei Technology Co. Ltd (Contract No: 4600000955)

\section{Author contributions:}

Conceptualization: GZ, DH

Methodology: GZ, DH, LW

Investigation: JL, PW, XY, YZ

Visualization: GZ, DH, LW

Funding acquisition: GZ, DH, JL, YZ, TEM

Project administration: JL, GZ, DH, AGA

Supervision: DZ, DH, LW, TEM, AGA 
Writing - original draft: DZ, DH, JX, CPG, JL

Writing - review \& editing: DZ, DH, CPG, TEM

Competing interests: Authors declare that they have no competing interests.

\section{Additional information}

The following are in the supplementary information.

Supplementary Text

Figs. $\mathrm{S} 1$ to $\mathrm{S} 4$

Tables S1 to S3 


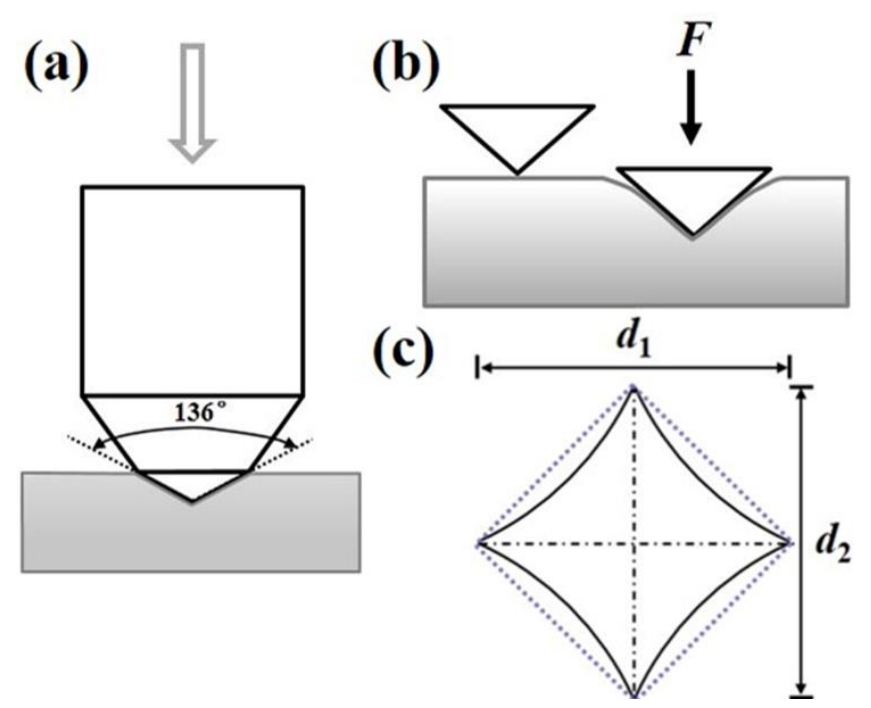

Fig. 1. Schematic pictures and process of the Vickers indenter exerting on the well-polished surface of a test sample. a Angle between the two opposite faces of a diamond pyramidal indenter is $136^{\circ}$. $\mathbf{b}$ Profile of a well-polished surface of test sample before and after exertion by an indenter. c Typical Vickers indentation produced by the diamond pyramidal indenter, the dotted lines represent ideal indentation, but the solid lines represent the actual indentation. 

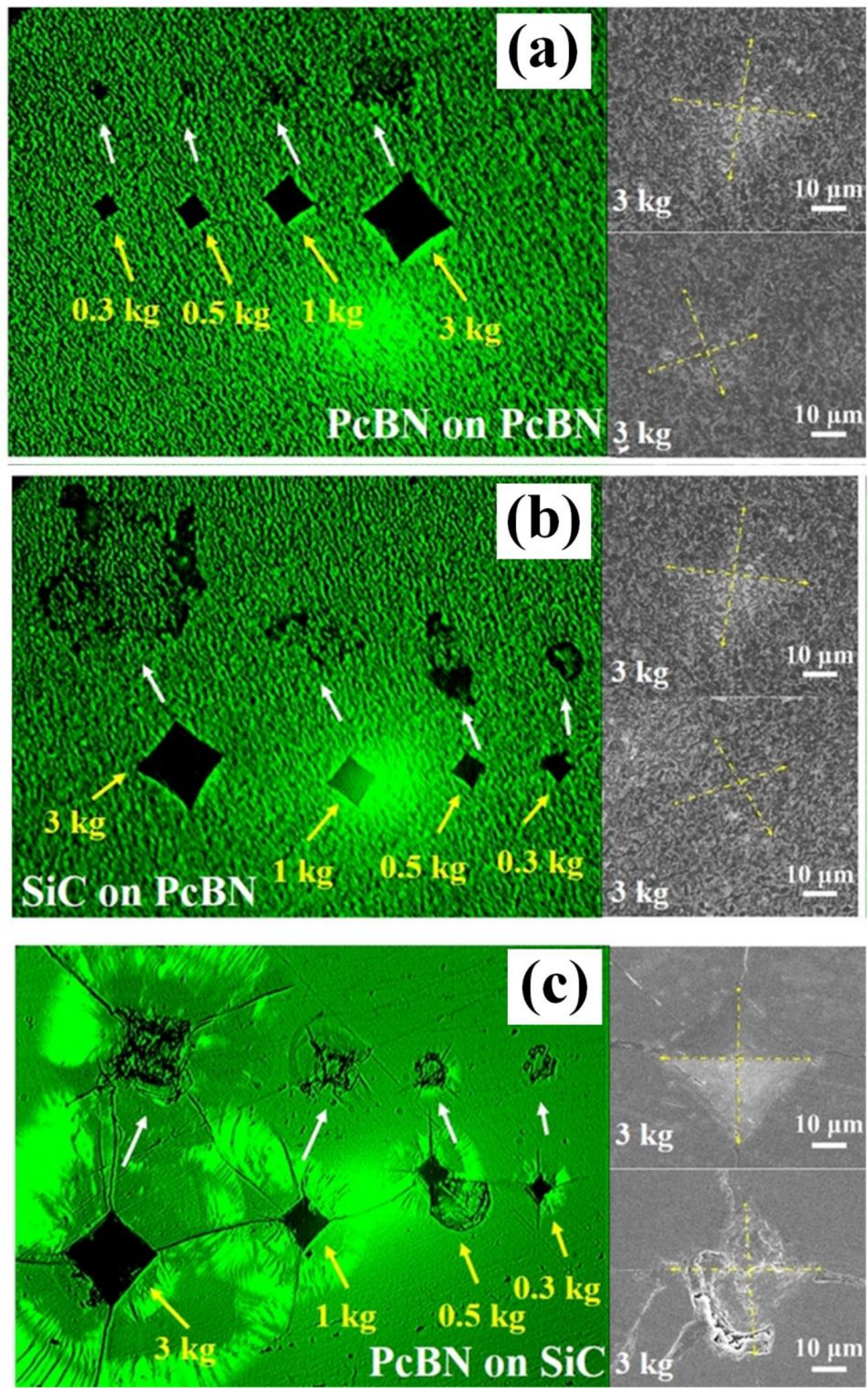

Fig. 2. Optical and SEM images of Vickers indentations of the test samples. Indentations of test samples produced by N-D indenter are marked with yellow arrows, and those produced by prepared indenters are marked with white arrows in optical images with various loads. The insert (above) in SEM image shows the indentation produced by N-D indenter and the 
insert (below) in SEM image shows the indentation produced by prepared indenter. a Both test sample and indenter are PcBN with load $3 \mathrm{~kg}$. b Test sample is $\mathrm{SiC}$ and indenter is PcBN with load $3 \mathrm{~kg}$. c Test sample is PcBN and indenter is $\mathrm{SiC}$ with load $3 \mathrm{~kg}$. 


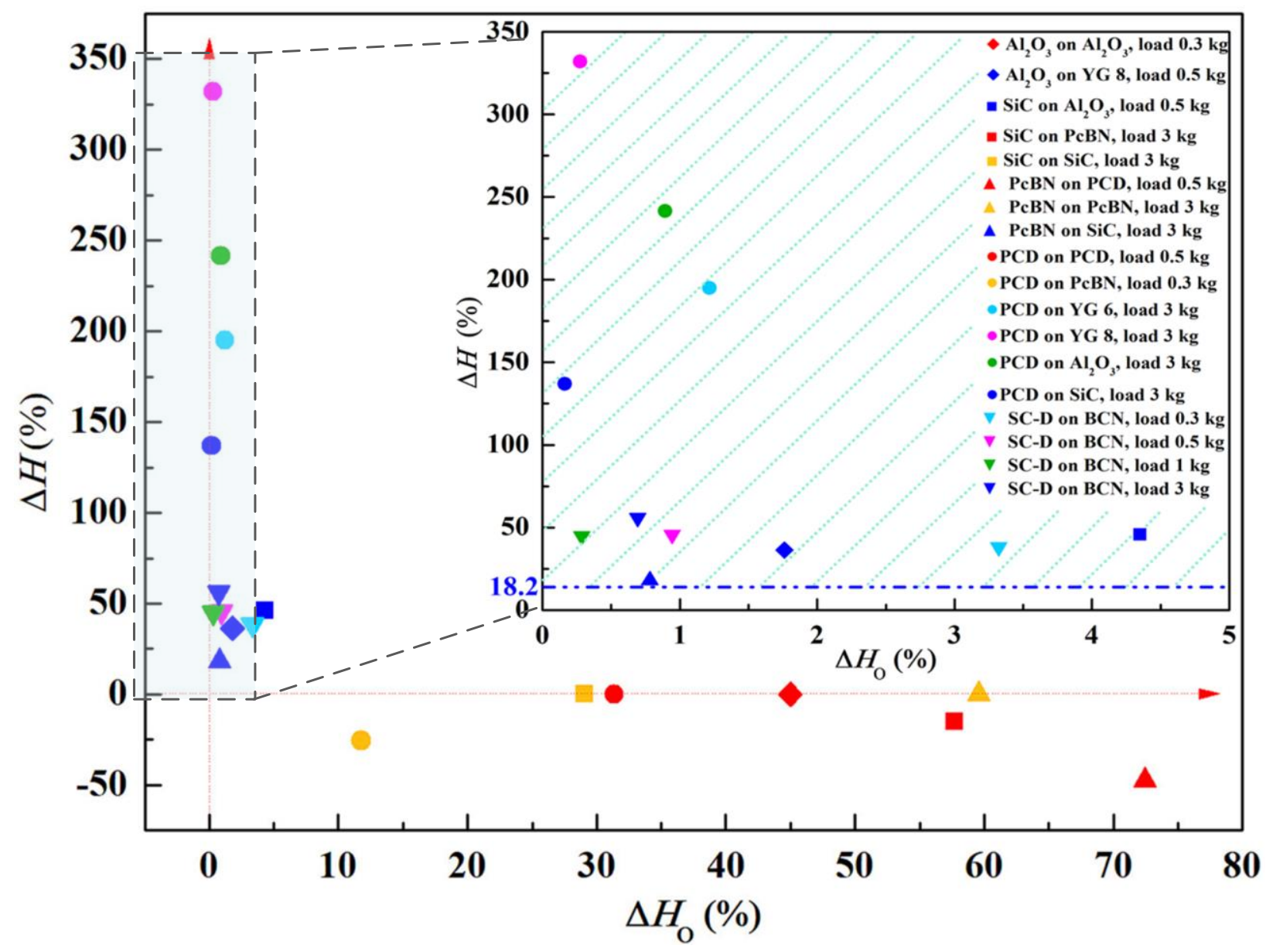

Fig. 3. Defining reliability. Relationship between relative percentage $(\Delta H)$ (hardness difference of the indenter vs test materials) and relative percentage $\left(\Delta H_{\mathrm{O}}\right)$ (measured value vs nominal value of test materials). Insert: closer-looked details of the relationship between $\Delta H$ and $\Delta H_{\mathrm{O}}$. Shaded and enlarged area is the reliable region. 


\section{Supplementary Files}

This is a list of supplementary files associated with this preprint. Click to download.

- NatComSupplementaryinformation.pdf 